\section{The Electron Microscopy Database: an Online Resource for Teaching and Learning Quantitative Transmission Electron Microscopy}

\author{
Paul M. Voyles \\ Department of Materials Science and Engineering, University of \\ Wisconsin, Madison, Madison, WI \\ voyles@engr.wisc.edu
}

\section{Introduction and Motivation}

Every spring, I teach a one-semester, graduate-level course on materials transmission electron microscopy (TEM). Thanks to the explosion of interest in nanotechnology, what was once a course primarily for metallurgists on imaging crystallographic defects and x-ray microanalysis now attracts a much broader audience. I have had students in the course from almost all the engineering departments at UW Madison (materials, chemical, mechanical, electrical, civil), from the basic sciences (physics, chemistry, geology), and from other departments (including one from Food Science!). The enrollment in the concurrent laboratory class on TEM operation is similar.

This diverse student body has two consequences. First, the students' background knowledge varies widely. Some have already taken a materials characterization course that included some TEM, but others barely know what a crystal structure is. I try to accommodate all comers with remedial reading materials and homework exercises. Second, the students' range of research interests virtually guarantees that someone will be interested in every technique available to modern TEM, be it imaging, diffraction, or spectroscopy. I try to cover almost everything, which makes for a busy semester.

The first two times I taught the course, one of its greatest deficiencies was a lack of high-quality examples for lectures and homework. It is one thing to hear about some imaging technique; it is something else entirely to see a top-quality example. The primary literature has many beautiful examples, but it proved less helpful than I expected for teaching. In addition to raising issues with distributing course materials freely without violating a journal's copyright, examples from the literature are often missing supporting, associated, or intermediate data. For example, HRTEM images do not always come with their power spectra, or defect images with the associated diffraction pattern. This data would arguably waste space in the journal, but it can be crucial for teaching.

The situation is worse for homework. Now that linear detectors like CCD cameras are ubiquitous, I believe that we should be treating the results of our microscopy observations as data, not pictures. Dealing quantitatively with data requires different practices for handling, processing, and measurement, and often requires different software tools, than dealing qualitatively with pictures. I emphasize quantitative methods in my class, and I think the best way to do that is to have students work with real, unprocessed, full bit-depth, full-resolution data sets on their homework assignments. That essentially excluded scans and digitizations from the primary literature as a source of examples.

That left my research. My research interests are the nanoscale structure of glasses and high-resolution imaging of defects in semiconductors and superconductors. I use only a subset of the techniques of modern TEM, on only a small subset of possible materials, so examples from my research did not meet the needs of my students. Even if I did have the time and expertise to obtain high-quality examples of all possible combinations of techniques and materials, I do not have local access to all the necessary instrumentation.

Discussions with colleagues at UW and elsewhere expanded my pool of examples, but also convinced me that many other TEM teachers are in the same boat. The result was the Electron Microscopy Database (EMdb), a repository for example datasets for teaching and learning TEM that is on the web at tem.msae. wisc.edu/emdb/. The goals of the EMdb are:

- to enhance instruction of graduate and undergraduate students in TEM by allowing them to work with real data that would be prohibitively difficult to acquire locally, either due to constraint of time and effort or lack of availability of specialized equipment;

- to encourage the use of quantitative methods in TEM by providing access to high-quality test data sets with suggested exercises; and

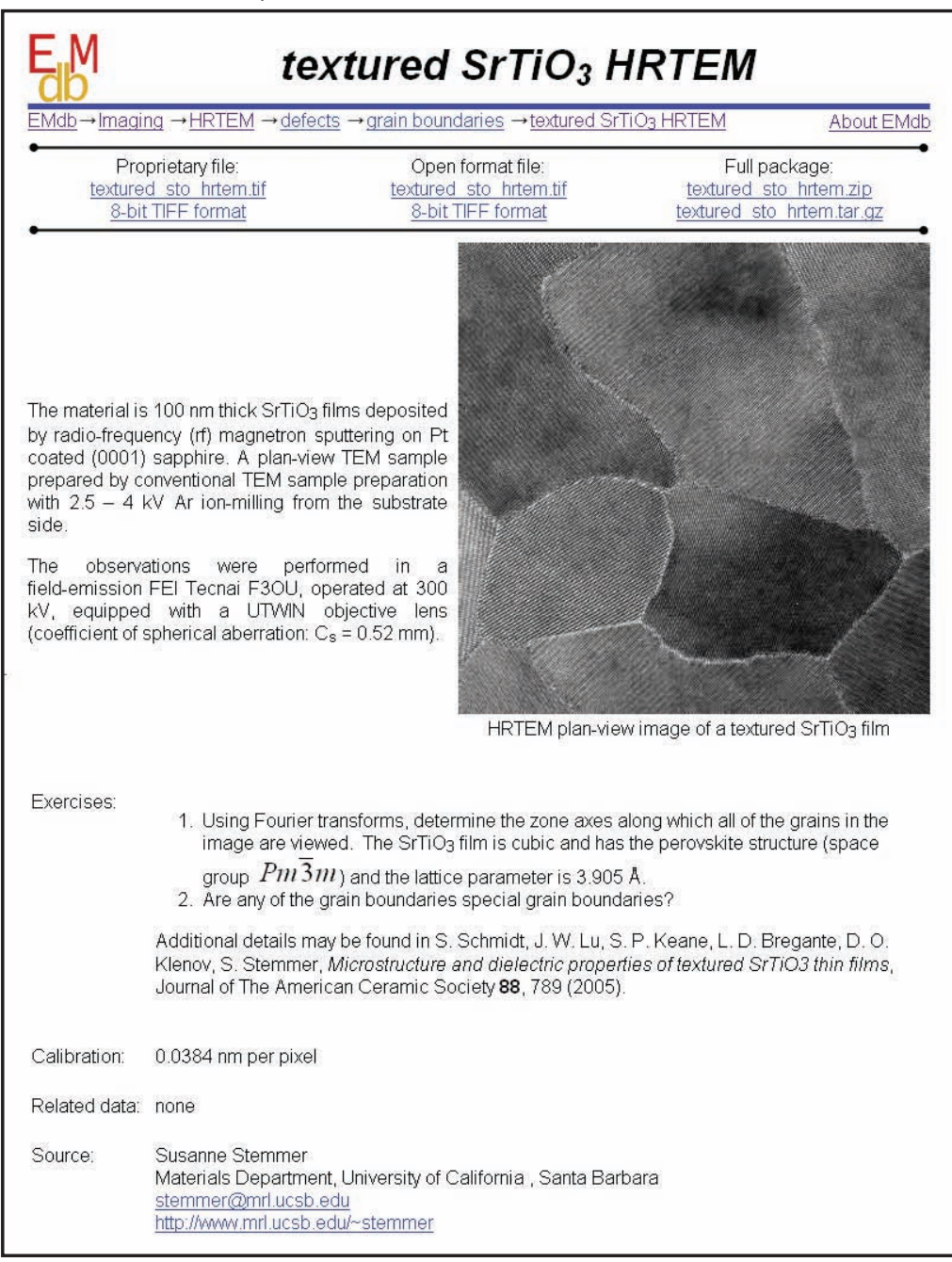

Figure 1: A typical EMdb record, as it appears on the Web. 


\section{MIR/A $\|_{\text {Schottky FE-SEM }}$}

\section{Value and Excellence in SEMs}

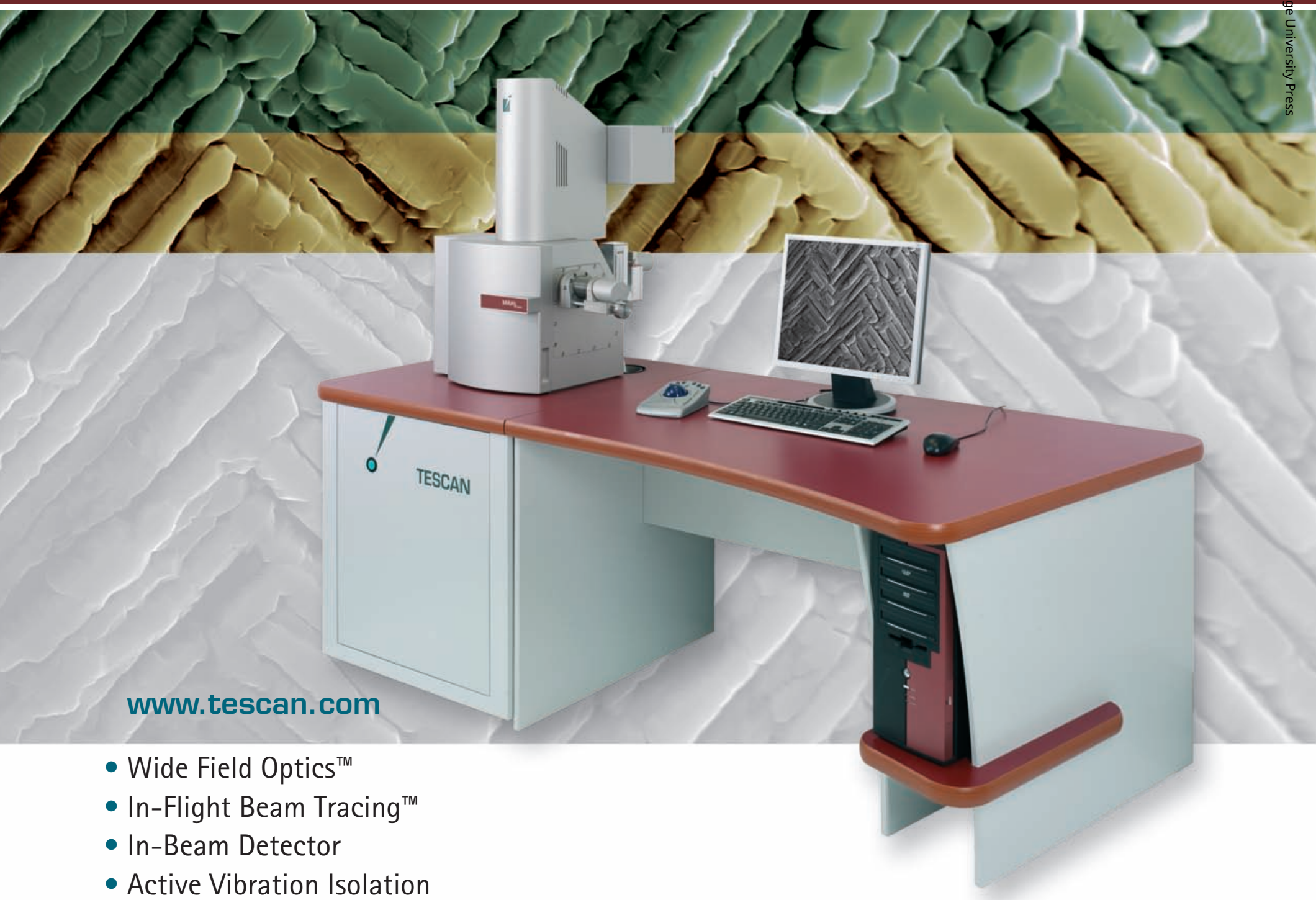

All as Tescan standard for the highest-quality imaging 